\title{
Preliminary observations of water masers associated with IRAS4A and IRAS4B
}

\author{
Kevin B. Marvel \\ American Astronomical Society, 2000 Florida Avenue, NW, Suite 400, \\ Washington, DC, 20009, USA \\ Mark Claussen \\ National Radio Astronomy Observatory, Array Operations Center, 1003 \\ Lopezville Road, Socorro, NM, 87801 USA \\ Alwyn Wootten \\ National Radio Astronomy Observatory Headquarters, 520 Edgemont \\ Road, Charlottesville, VA, 22903-2475, USA
}

\begin{abstract}
We present preliminary observations of water masers associated with IRAS4, a pair of young stellar objects in the NGC 1333 star forming region. The masers are quite strong and spatially distinct. Proper motion observations have been obtained from various epochs of observation typically separated by about three weeks. IRAS4A consists of two regions of maser activity separated by about $114 \mathrm{AU}$ along a position angle of 138 degrees. Expansion of about $68 \mathrm{~km} / \mathrm{s}$ is detected. IRAS4B consists of two linear emission regions separated by about 175 AU on a 150 degree position angle. The linear features are about $15 \mathrm{AU}$ in length and about 2 AU thick. They are expanding away from each other at about $70 \mathrm{~km} / \mathrm{s}$.
\end{abstract}

\section{Introduction}

IRAS 4 is located in the star forming cloud NGC1333, located about 350 pc from the Earth. It is a well studied region and inherently interesting optically. It displays emission, reflection and absorption features along with $\mathrm{HH}$ objects and many young stars. A striking series of images has been created by Bally \& Reipurth (2000).

Not only is IRAS 4 a multiple system (Jennings et al. 1987), but it is multiply multiple (Lay et al. 1995). The two components are separated by about $11,000 \mathrm{AU}$ along a position angle of $135^{\circ}$. Age estimates vary, but $4 \mathrm{~A}$ is assumed to be about 500,000 years old. Both sources are cold, with temperatures of about $30 \mathrm{~K}$.

Blake et al. (1995) detected a well-collimated molecular outflow from IRAS $4 \mathrm{~A}$ as well as a more compact outflow from IRAS $4 \mathrm{~B}$. Interestingly, the outflow from IRAS 4A is aligned northeast-southwest on large scales, but transitions to a north-south alignment close to the driving source. Lay et al. (1995) predicted 
that $4 \mathrm{~A}$ and $4 \mathrm{~B}$ are multiple systems using the single baseline of the JCMT-CSO interferometer. Subsequent SCUBA observations by Smith et al. (2000) have confirmed this interferometeric observation. Mass estimates from the submillimeter observations (Smith et al. 2000) give values of $11 \mathrm{M}_{\odot}$ for $4 \mathrm{~A}$ (which appears as an extended disk-like object in the SCUBA image), 6 and $2 \mathrm{M}_{\odot}$ for the components of IRAS $4 \mathrm{~B}$ and $3 \mathrm{M}_{\odot}$ for a previously unnoticed component IRAS 4C, which may not be dynamically linked to the 4A-4B system.

Akeson et al. (1997) imaged the polarized emission towards IRAS 4 and suggested that this emission arises from magnetically aligned dust grains. The derived magnetic field stucture is aligned roughly parallel with the large-scale outflow direction of NE-SW. This polarization is also observed at $800 \mu \mathrm{m}$ (Minchin et al. 1995). These observations are consistent with the idea that the polar magnetic field of these objects is aligned with the outflow.

Claussen et al. (1996) monitored the water maser emission monthly using large single dish telescopes and showed that the masers associated with IRAS $4 \mathrm{~A}$ and $4 \mathrm{~B}$ were highly variable (in addition to a large number of other interesting sources). Although unable to determine which of the two sources the maser emission was originating from due to insufficient resolution, the highly variable nature of the source and two distinct velocity groupings hinted that there were masers were associated with both sources.

We have subsequently observed the water masers of both IRAS 4A and 4B with the Very Long Baseline Array and present preliminary results from these observations here.

\section{Observations}

Using the ten antennas of the VLBA, we have imaged the water masers of IRAS $4 \mathrm{~A}$ and $4 \mathrm{~B}$. We used a standard frequency setup and reduced the data using the AIPS. After standard delay and rate correction, we select a strong maser feature to map. If the structure of the feature is relatively simple and the source is strong, we iteratively self-calibrate and apply the resultant gain corrections to the entire set of observed channels. In practice this mapping method is reliable and produces high-quality images, but absolute position information is lost in the process.

The masers of IRAS 4A (Figure 1) are distributed in two clumps, which are separated by about $114 \mathrm{AU}$. The clumps are expanding away from each other and display an expansion of about $68 \mathrm{~km} / \mathrm{s}$. Unlike other low-mass sources (e.g. IRAS 05413-0104) the number of masers detected is quite low, and no bow-shock structures are seen.

The masers of IRAS 4B (Figure 2) are also distributed in two clumps, however they are aligned in linear filaments. The southern filament forms an arc-like structure that is about $15 \mathrm{AU}$ in size and less than $2 \mathrm{AU}$ thick. The northern feature is also linear, but is slightly longer (5 mas) and thicker (1-3 mas).

Although exact one-to-one matching of the maser components is not yet complete, it appears the two regions are separating at about $70 \mathrm{~km} / \mathrm{s}$ along a position angle of $138^{\circ}$, exactly the same as the position angle of IRAS $4 \mathrm{~A}$ and $4 \mathrm{~B}$ and the IRAS 4B binary system. 


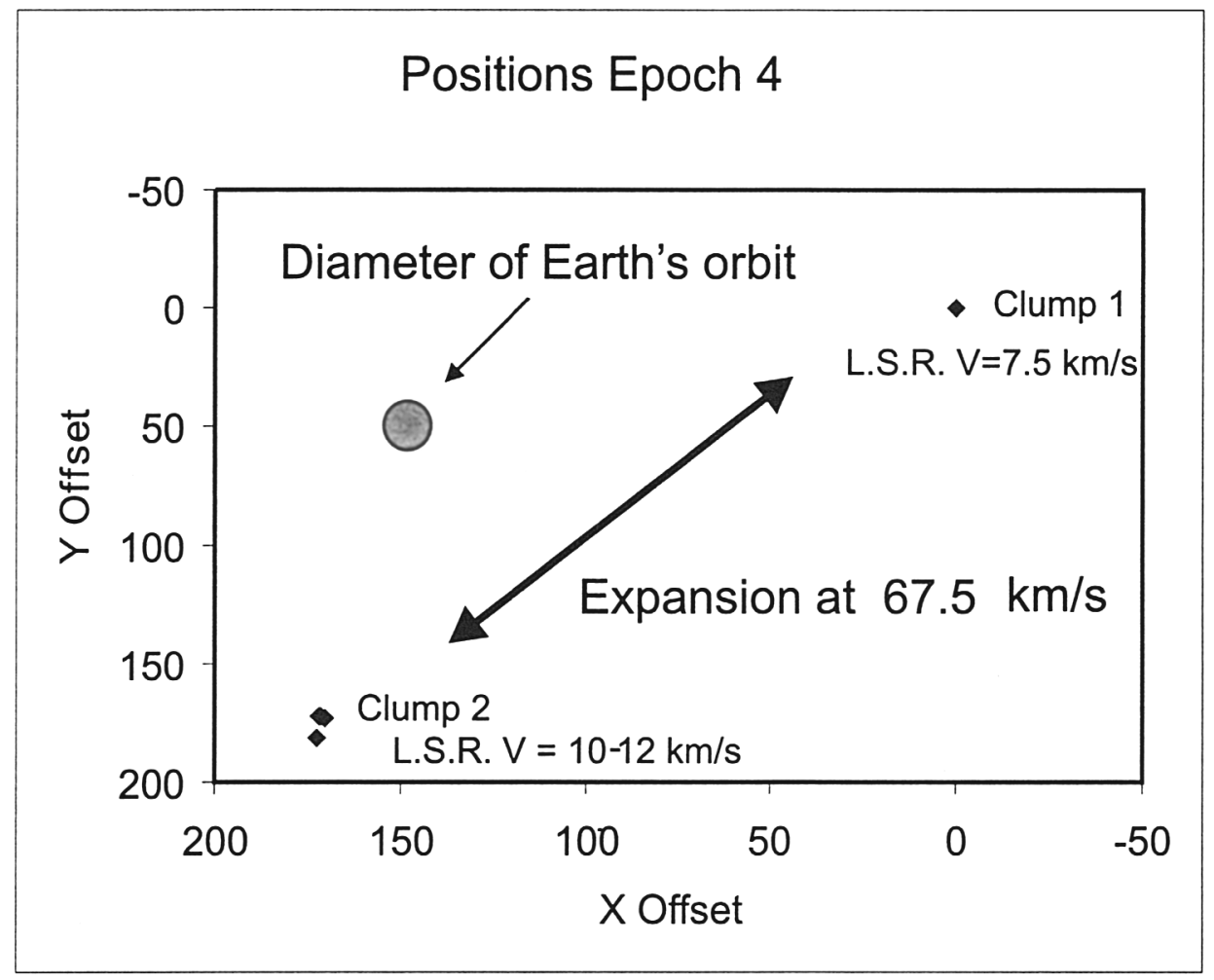

Figure 1. A summary graphic showing the water maser distribution and observed proper motions for IRAS $4 \mathrm{~A}$

\section{Conclusion}

The IRAS 4A observations are not detailed enough to tell us much about their relationship with the underlying driving source. However, the masers of IRAS 4B are interestingly distributed (being perpendicular to the large-scale outflow and parallel with the large-scale disk-like structure of IRAS 4B itself seen in the submillimeter). Despite this apparent alignment with the IRAS 4B system, the proper motions currently measured rule out the possibility of the masers existing in a disk. They are simply too high for the measured mass of the IRAS $4 \mathrm{~B}$ system or its components.

Subsequent reduction of the remaining epochs of observations and analysis should help untangle the distribution and kinematics of the IRAS 4B water masers. However, absolute position measurements of the masers and their driving source are truly needed. Perhaps the SMA or ALMA combined with phasereferenced VLBA observations will lead to a complete picture of this interesting young star.

Acknowledgments. K.B.M. wishes to thank the Brazilian embassy in D.C. for ensuring that A.W. would be the person presenting this talk in Angra dos Reis. 

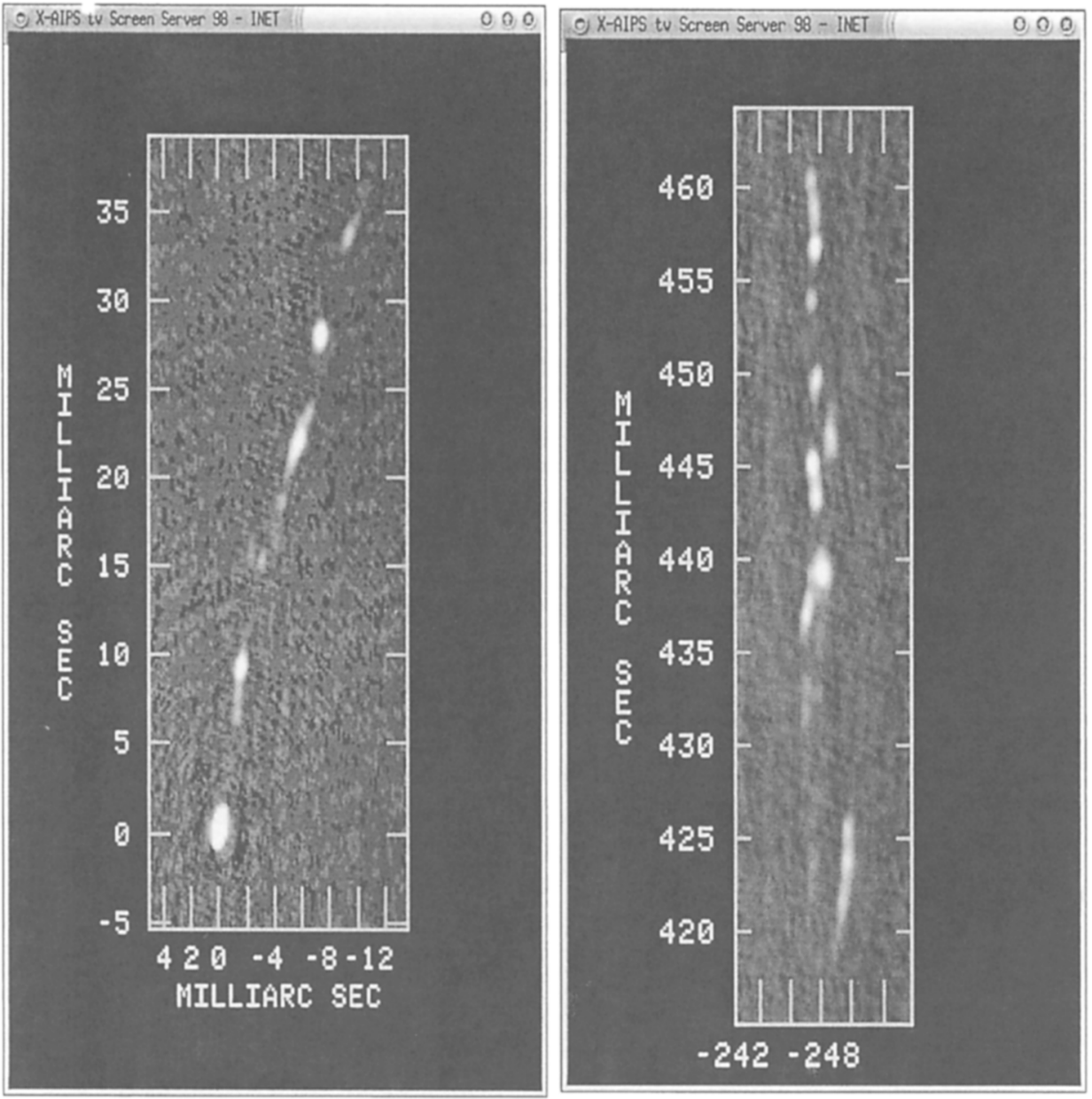

Figure 2. The southern water maser filament associated with IRAS 4B (left) and the norther water maser filament (right). They are both about 15 AU long.

\section{References}

Akeson, R. L. and Carlstrom, J. E., 1997, ApJ, 491, 254 
Bally, J. and Reipurth, B., 1999, ApJ, 546, 299

Blake, G. A., Sandell, G., van Dishoeck, E. F., Groesbeck, T. D., Mundy, L. G., \& Aspin, C., 1995, ApJ, 441, 689

Claussen, M. J., Wilking, B. A., Benson, P. J., Wootten, A., Myers, P. C., \& Tereby, S., 1996, ApJS, 106, 111

Jennings, R. E., Cameron, D. M. H., Cudlip, W.., and Hirst, C. J., 1987, MNRAS. 226,461

Lay, O. P., Carlstrom, J. E., and Hills, R. E., 1995, ApJ, 452, L73

Minchin, N.R., Sandell, G., and Murray, A.G., 1995, å, 293, 61

Smith, K. W., Bonnell, I. A., Emerson, J. P., Jenness, T., 2000, MNRAS, 319, 991

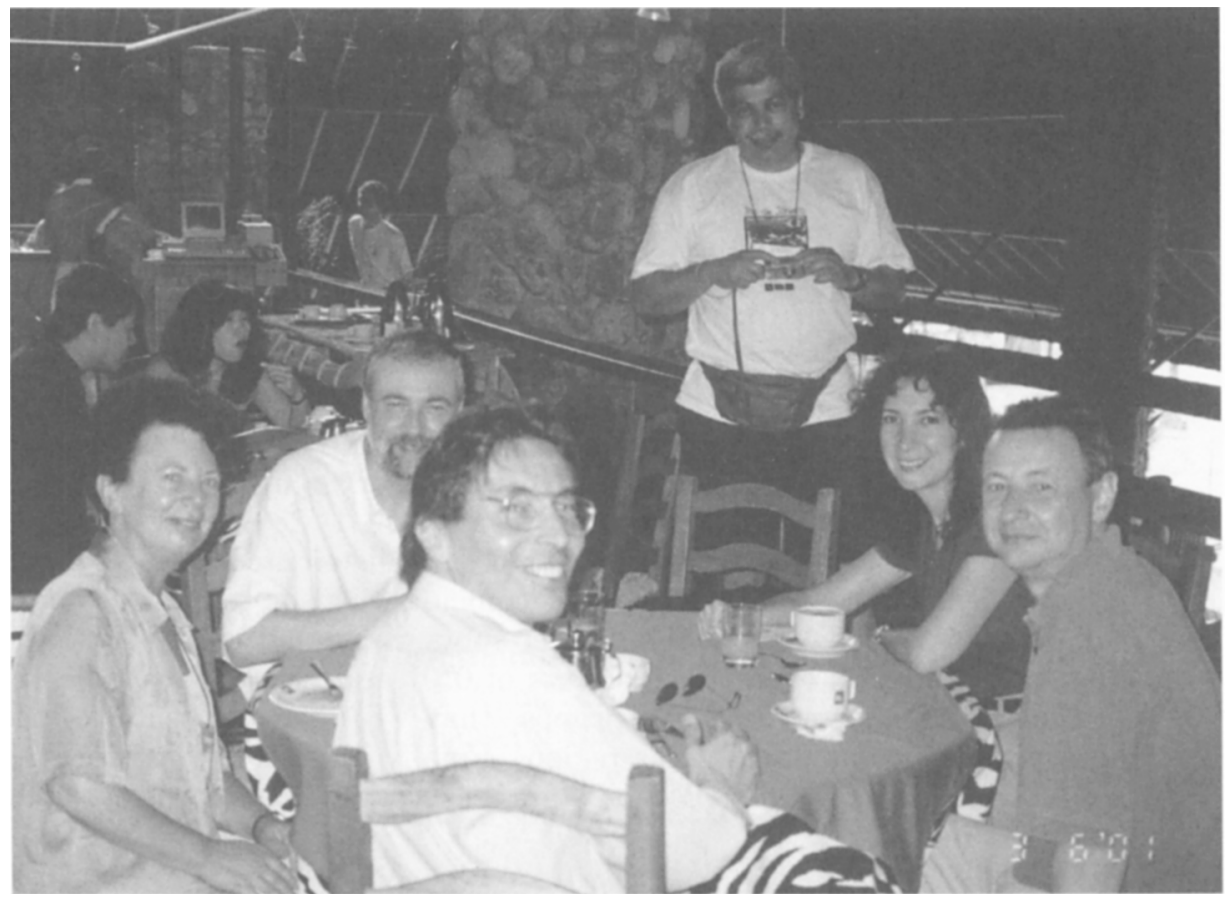

